Research Paper: Pathology

\title{
Interferon gamma peptidomimetic targeted to interstitial myofibroblasts attenuates renal fibrosis after unilateral ureteral obstruction in mice
}

\author{
Fariba Poosti ${ }^{1,5}$, Ruchi Bansal², Saleh Yazdani², Jai Prakash², Leonie Beljaars ${ }^{3}$, \\ Jacob van den Born ${ }^{4}$, Martin H. de Borst ${ }^{4}$, Harry van Goor ${ }^{1}$, Jan-Luuk Hillebrands ${ }^{1, *}$ \\ and Klaas Poelstra ${ }^{3, *}$ \\ ${ }^{1}$ Department of Pathology and Medical Biology, Division of Pathology, University Medical Center Groningen, University of \\ Groningen, Groningen, The Netherlands \\ 2 Department of Biomaterials Science and Technology, Division of Targeted Therapeutics, MIRA Institute, University of \\ Twente, Enschede, The Netherlands \\ ${ }^{3}$ Department of Pharmacokinetics, Toxicology and Targeting, University of Groningen, Groningen, The Netherlands \\ ${ }^{4}$ Department of Internal Medicine, Division of Nephrology, University Medical Center Groningen, University of Groningen, \\ Groningen, The Netherlands \\ ${ }^{5}$ Department of Microbiology and Immunology, Laboratory of Molecular Immunology, Rega Institute, KU Leuven, Belgium \\ * Share senior authorship \\ Correspondence to: Jan-Luuk Hillebrands, email: j.l.hillebrands@umcg.nl \\ Keywords: drug targeting, fibrosis, interferon gamma, kidney, myofibroblast, Pathology Section \\ Received: March 07, $2016 \quad$ Accepted: June 20, $2016 \quad$ Published: August 05, 2016
}

\section{ABSTRACT}

Renal fibrosis cannot be adequately treated since anti-fibrotic treatment is lacking. Interferon- $y$ is a pro-inflammatory cytokine with anti-fibrotic properties. Clinical use of interferon- $y$ is hampered due to inflammation-mediated systemic side effects. We used an interferon-Y peptidomimetic (mimy) lacking the extracellular IFNyReceptor recognition domain, and coupled it to the PDGF $\beta R$-recognizing peptide BiPPB. Here we tested the efficacy of mimy-BiPPB (referred to as "Fibroferon") targeted to PDGF $\beta$ R-overexpressing interstitial myofibroblasts to attenuate renal fibrosis without inducing inflammation-mediated side effects in the mouse unilateral ureter obstruction model.

Unilateral ureter obstruction induced renal fibrosis characterized by significantly increased a-SMA, TGF $\beta 1$, fibronectin, and collagens I and III protein and/or mRNA expression. Fibroferon treatment significantly reduced expression of these fibrotic markers. Compared to full-length IFNy, anti-fibrotic effects of Fibroferon were more pronounced. Unilateral ureter obstruction-induced lymphangiogenesis was significantly reduced by Fibroferon but not full-length IFNy. In contrast to full-length IFNy, Fibroferon did not induce IFNy-related side-effects as evidenced by preserved low-level brain MHC II expression (similar to vehicle), lowered plasma triglyceride levels, and improved weight gain after unilateral ureter obstruction.

In conclusion, compared to full-length IFNy, the IFNy-peptidomimetic Fibroferon targeted to PDGF $\beta$ R-overexpressing myofibroblasts attenuates renal fibrosis in the absence of IFNy-mediated adverse effects. 


\section{INTRODUCTION}

Patients with chronic kidney disease (CKD) are at increased risk of progression to end-stage renal disease (ESRD). Virtually all forms of CKD are characterized by significant amounts of renal fibrosis [1, 2]. Renal interstitial fibrosis is the final common process observed in various kidney diseases that leads to renal failure, and is associated with tubular atrophy and dilation, interstitial matrix deposition, accumulation of interstitial fibroblasts, and inflammatory cell infiltration [3]. There is still no effective therapy to halt renal fibrogenesis, and to prevent progression to ESRD of which the latter requires renal replacement therapy eventually [4]. Among the characteristic changes in the kidney during development of fibrosis, interstitial extracellular matrix (ECM) deposition is the key step. Interstitial fibroblasts are the predominant source of ECM constituents. Therefore, dampening of interstitial fibroblast activation and subsequent prevention of differentiation into ECM-producing myofibroblasts, is an attractive approach to attenuate development of renal interstitial fibrosis [5-7].

Interferon- $\gamma($ IFN $\gamma)$ is a cytokine primarily produced by $\mathrm{T}$ cells and natural killer (NK) cells. It is a pleiotropic cytokine with anti-viral, anti-bacterial, anti-tumor, proinflammatory but also anti-fibrotic activities [8]. Because of the latter, IFN $\gamma$ is a potential effective biological to treat or prevent renal fibrosis [9-12]. However, its potential therapeutic value in fibrosis has been limited so far due to the systemic side effects (because of widespread IFN $\gamma$ receptor [IFN $\gamma \mathrm{R}]$ expression) and rapid renal clearance [13-15]. In order to overcome these problems as well as to attain high concentrations in the target cells, cell-specific targeting via receptor-mediated uptake of biologicals is an attractive approach.

Activated fibroblasts in fibrotic tissue are characterized by increased platelet-derived growth factor receptor-beta (PDGFßR) expression [16, 17], and this prompted us to use the PDGF $\beta R$ for receptor-mediated uptake of biologicals. To do so, pegylated full length IFN $\gamma$ was conjugated to PDGF $\beta$ R-recognizing peptide (PPB) [18] and by using this conjugate (i.e. PPB-PEG-IFN $\gamma$ ) we were able to demonstrate anti-fibrotic effects in the $\mathrm{CCl}_{4}$-induced liver fibrosis mouse model [19] and antitumorigenic effects by targeting stromal cells in the B16 melanoma tumor mouse model [20]. Recently we used the same targeting approach as proof of concept in the mouse unilateral ureteral obstruction (UUO) model of renal fibrosis in which anti-fibrotic effects of the PPB-PEGIFN $\gamma$ conjugate were demonstrated [12]. Despite the antifibrotic effects of PPB-PEG-IFN $\gamma$, systemic side effects were mildly reduced but remained still present compared to full length IFN $\gamma$ as evidenced by increased brain MHC class II expression. This side effect was most likely due to binding of the full length IFN $\gamma$ to the ubiquitously expressed IFN $\gamma$ R.
To overcome the problem of IFN $\gamma$ R-mediated systemic side effects we recently designed a new IFN $\gamma$ based biological which lacks the amino-terminal IFN $\gamma \mathrm{R}$ binding sequence $[21,22]$. This IFN $\gamma$-peptidomimetic $(\operatorname{mim} \gamma)$ was conjugated to the bicyclic platelet-derived growth factor receptor-beta recognizing peptide (BiPPB) for targeting to PDGF $\beta$ R-expressing cells (schematically depicted in Figure 1a). The mim $\gamma$-BiPPB was recently renamed "Fibroferon" [23]. Here we tested the hypothesis that compared to the previously tested non-targeted full-length IFN $\gamma$ [12], specific delivery of Fibroferon to PDGF $\beta$ R-overexpressing interstitial myofibroblasts attenuates renal fibrosis and reduces inflammationmediated side-effects in the mouse UUO model.

\section{RESULTS}

\section{PDGF $\beta R$ expression is increased on interstitial myofibroblasts in UUO mouse kidneys}

We first analyzed PDGF $\beta R$ expression in mouse sham and fibrotic (UUO) kidneys. Confirming our previous data, fibrosis in UUO is characterized by increased interstitial PDGF $\beta \mathrm{R}$ expression as revealed by increased mRNA expression (Figure 2a) and protein expression (based on immunohistochemistry) (Figure 2b). $\mathrm{w}$

\section{Fibroferon reduces renal fibroblast activation}

In order to determine whether Fibroferon reduces renal fibroblast activation in vivo, $\alpha$-SMA expression was determined at both mRNA and protein level. As shown in Figure 3a, UUO (receiving subsequent treatment with vehicle) resulted in increased $(p<0.001) \alpha$-SMA mRNA expression compared to sham controls. $\alpha$-SMA mRNA expression after treatment with Fibroferon and non-targeted full length IFN $\gamma$ was significantly lower compared with vehicle-treated UUO mice $(p<0.05$ vs. vehicle), and similar to sham-operated (no UUO) mice. Immunohistochemistry confirmed increased $\alpha$-SMA expression in UUO kidneys (vehicle treatment, $p<0.01$ vs. sham, Figure 3b,c). Representative photomicrographs of $\alpha$-SMA staining are shown in Figure 3b. Quantitative analysis revealed significantly reduced $\alpha$-SMA protein expression after Fibroferon treatment when compared to vehicle treatment $(p<0.05 v s$. vehicle, Figure $3 c)$.

Since TGF $\beta 1$ is known to induce $\alpha$-SMA expression in fibroblasts [24] we also analyzed TGF $\beta 1$ mRNA expression in sham and UUO kidneys. UUO (vehicle) significantly increased renal TGF $\beta 1$ expression $(p<0.01$ vs. sham), which was not prevented by Fibroferon or IFN $\gamma$ treatment (Figure 3d). 
a

$\underbrace{}_{\text {PDGF }}$ myofibroblast

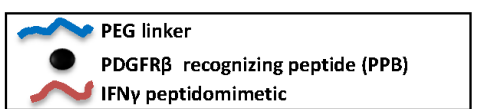

with mimy-BiPPB

(i.e. with Fibroferon)

no mimy-BiPPB

(i.e. no Fibroferon)
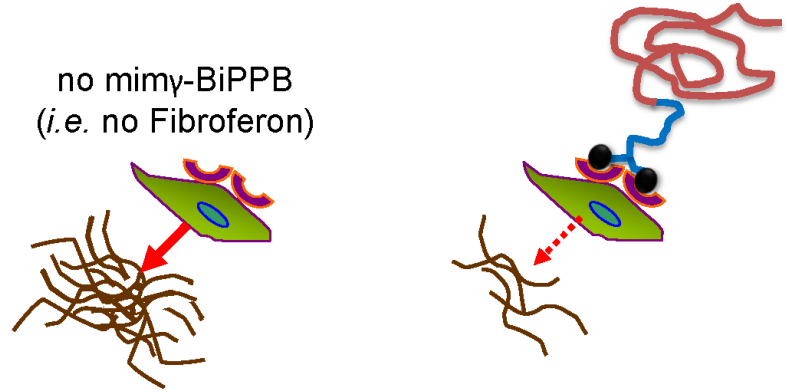

b

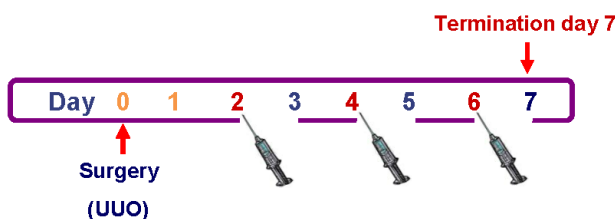

Figure 1: Schematic representation of the structure of the targeted IFN $\gamma$ peptidomimetic Fibroferon (mim $\gamma$-BiPPB) and its binding to PDGFRß-expressing myofibroblasts (a), and the in vivo treatment regimen in the mouse unilateral ureter obstruction (UUO) model (b).

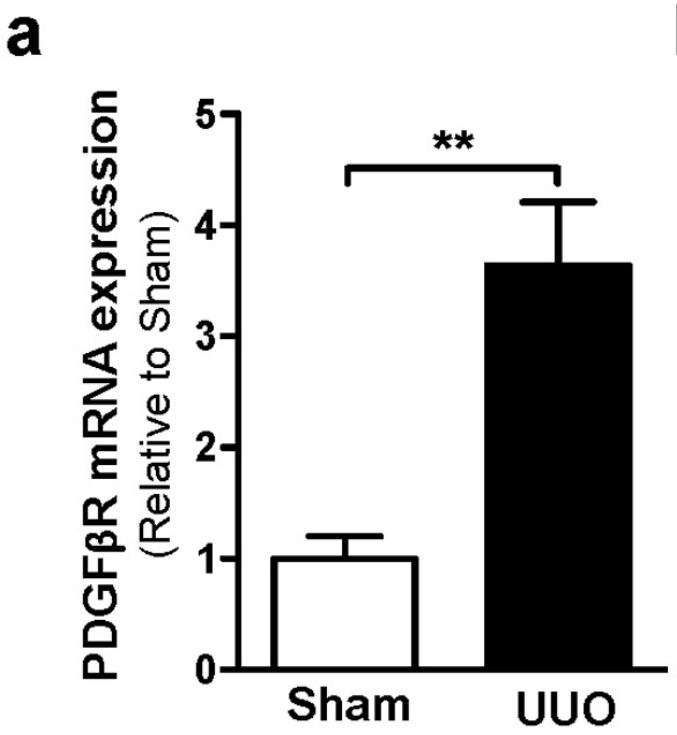

b

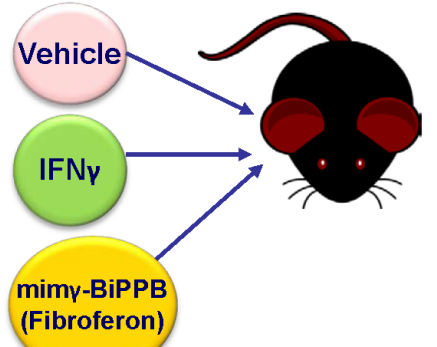

(Fibroferon)

-

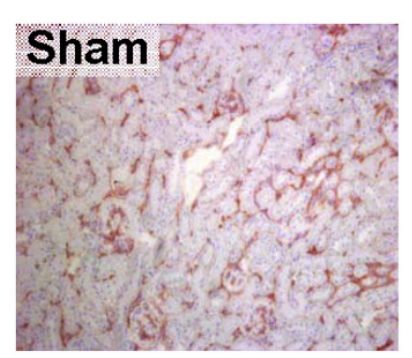

Figure 2: UUO increases PDGFßR expression on mRNA (a) and protein (b) expression level. a. Relative gene expression of PDGFßR in UUO (vehicle-treated) and sham-operated control kidneys at day 7 post surgery. ${ }^{* *} p<0.01 v s$. sham. Bars represent mean \pm SEM of 5-6 mice per group. b. Representative photomicrographs (200x) of kidney sections from sham-operated and vehicle-treated UUO mice stained for PDGFßR. 


\section{Fibroferon reduces extracellular matrix (ECM) deposition in UUO kidneys}

Next we analyzed the effects of Fibroferon on ECM constituent expression using immunohistochemistry. Clear differences in protein expression were observed when comparing the different treatment groups (Figure 4). In UUO (vehicle) significantly increased protein expression levels for interstitial fibronectin (Figure 4a), collagen I (Figure 4b), and collagen III (Figure 4c) were observed 7 days post-UUO compared to sham-operated mice (all $p<0.01 v s$. sham). Compared to vehicle, only treatment with Fibroferon resulted in significantly reduced fibronectin (Figure $4 \mathrm{a}, p \leq 0.05$ ), collagen I (Figure $4 \mathrm{~b}, p$ $<0.01$ ), and collagen III (Figure $4 \mathrm{c}, p \leq 0.05$ ) expression. Protein expression in non-targeted full length IFN $\gamma$ did not statistically differ from vehicle-treated mice. Compared with non-targeted full length IFN $\gamma$, these data indicate that Fibroferon is more effective in reducing ECM constituent expression.

\section{Effects of Fibroferon on renal inflammation in UUO}

IFN $\gamma$ exerts systemic pro-inflammatory effects but possibly also local intra-renal inflammatory responses. To analyze the latter, we determined whether treatment with non-targeted full length IFN $\gamma$ and Fibroferon resulted in different intra-renal inflammatory responses as determined by $\mathrm{qPCR}$ analyses, and $\mathrm{CD}^{+} \mathrm{T}$ cell infiltration. As shown in Suppl. Figure 1, compared to sham-operated mice, UUO (with subsequent vehicle treatment) resulted in significantly increased MCP-1 (a), TNF- $\alpha$ (b), IL-1 $\beta$ (c) and IL-6 (d) mRNA expressions (all $p<0.01 v s$. sham). Although Fibroferon tended to reduce, and reciprocally non-targeted full length IFN $\gamma$ tended to increase proinflammatory cytokine production ( $v s$. vehicle), no statistically significant differences were observed.

UUO (vehicle) significantly increased renal MHC class II expression that was reduced after Fibroferon treatment when compared with non-targeted full length IFN $\gamma$ (Suppl. Figure 1e). This might be explained by increased IFN $\gamma$-induced macrophage activation rather than macrophage influx (Suppl. Figure 1f). Additionally, UUO resulted in increased $\mathrm{CD}^{+} \mathrm{T}$ cell influx as determined by immunohistochemistry ( $p<0.01 v s$. sham) that was reduced by mim $\gamma$-BiPPB (58\% reduction) and nontargeted full length IFN $\gamma$-treated mice ( $42 \%$ reduction) (Suppl. Figure 1g).

\section{Fibroferon reduces lymphangiogenesis}

New lymph vessel formation (lymphangiogenesis) has been shown in different renal disease models, including UUO [25]. This led us to hypothesize that UUOinduced renal lymphangiogenesis might be attenuated following Fibroferon treatment. Compared to sham, UUO (vehicle treatment) indeed increased numbers of podoplanin ${ }^{+}$-lymph capillaries $(p<0.01 v s$. sham) which were significantly lower in Fibroferon-treated $(p \leq 0.05$ $v s$. vehicle), but not in non-targeted full length IFN $\gamma$ treated mice (Figure 5a). Depending on the time after UUO induction, both angiogenesis (increase in capillary density) and capillary rarefaction has been described [26]. We analyzed the effect of Fibroferon and non-targeted full length IFN $\gamma$ on peritubular capillary density. As shown in Figure 5b, UUO (vehicle) was associated with significantly increased numbers of $\mathrm{CD} 31^{+}$peritubular capillaries $(p<0.01 v s$. sham), which was not reduced by Fibroferon. Photomicrographs shown in Figure 5a and $5 \mathrm{~b}$ display representative photos of podoplanin ${ }^{+}$lymph capillaries and $\mathrm{CD} 31^{+}$peritubular capillaries in UUO (vehicle, Fibroferon, and non-targeted full length IFN $\gamma$ treatment) and sham kidneys, respectively.

\section{Fibroferon treatment is accompanied by less systemic side effects}

To assess whether treatment with Fibroferon reduces systemic side effects compared with non-targeted full length IFN $\gamma$, brain MHC class II mRNA expression (as a measure of IFN $\gamma$-induced microglia activation) and plasma triglyceride levels (as a measure of IFN $\gamma$-induced lypolysis in adipose tissue) were determined. As shown in Figure $6 \mathrm{a}$, in line with our previous observations $[12,19$, 21] treatment with non-targeted full length IFN $\gamma$ caused significant $(p<0.01)$ up-regulation of brain MHC class II expression. In contrast, upon Fibroferon treatment brain MHC class II expression was similar to vehicle-treatment. Compared with non-targeted full length IFN $\gamma$, Fibroferon treatment significantly reduced plasma triglyceride levels $(p<0.05$, Figure $6 \mathrm{~b})$. Compared with vehicle, plasma triglyceride levels were not significantly increased after non-targeted full length IFN $\gamma$ treatment (not shown), which might be explained by the fact that we used nonpegylated full length IFN $\gamma$. Finally, we determined weight loss and recovery after induction of UUO in the various treatment groups. As shown in Figure 6c, mice receiving wFibroferon displayed less weight loss and accelerated weight gain after UUO when compared to non-targeted full length IFN $\gamma$ treated mice, which might be explained either by attenuating the renal damage and/or by reducing side effects in mice receiving Fibroferon.

\section{DISCUSSION}

In this study we demonstrate pronounced antifibrotic effects of targeted IFN $\gamma$ peptidomimetic to PDGF $\beta R$-expressing cells without identifiable systemic 
a

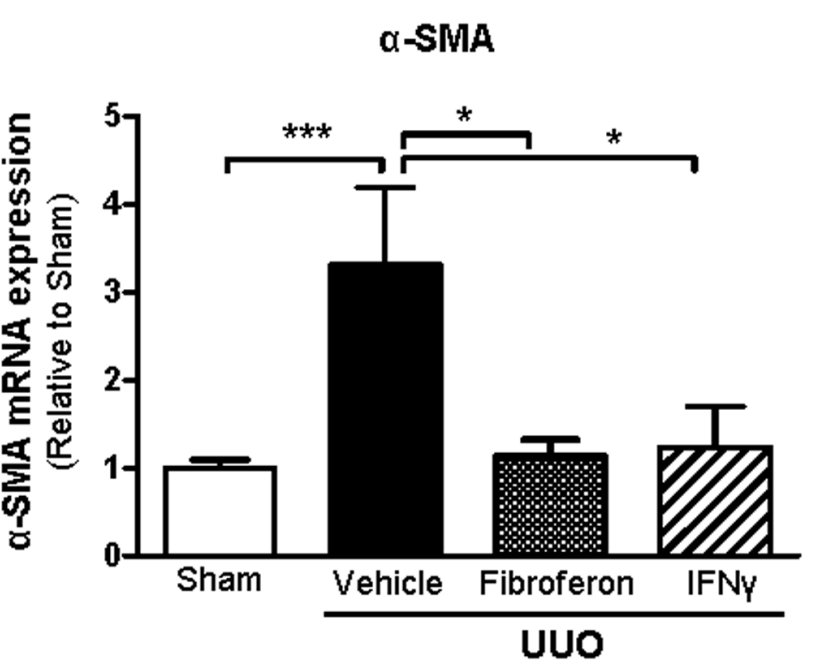

b
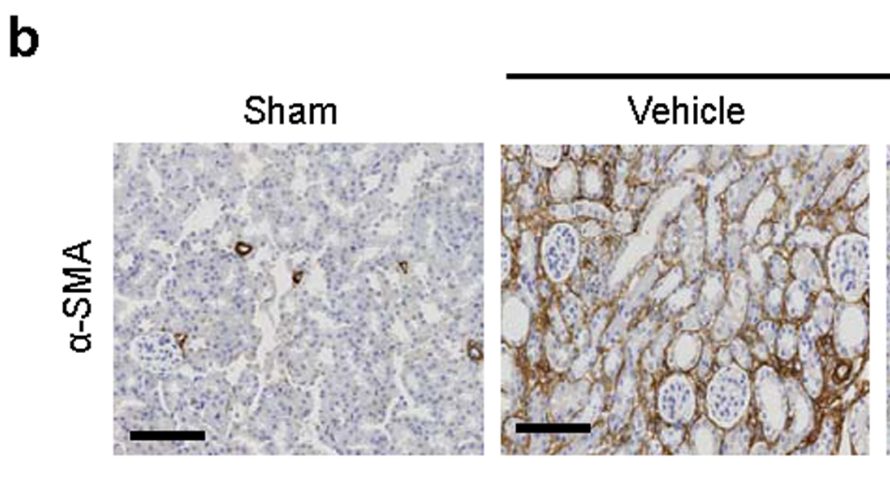

UUO

C

๙-SMA

d

TGF $\beta 1$
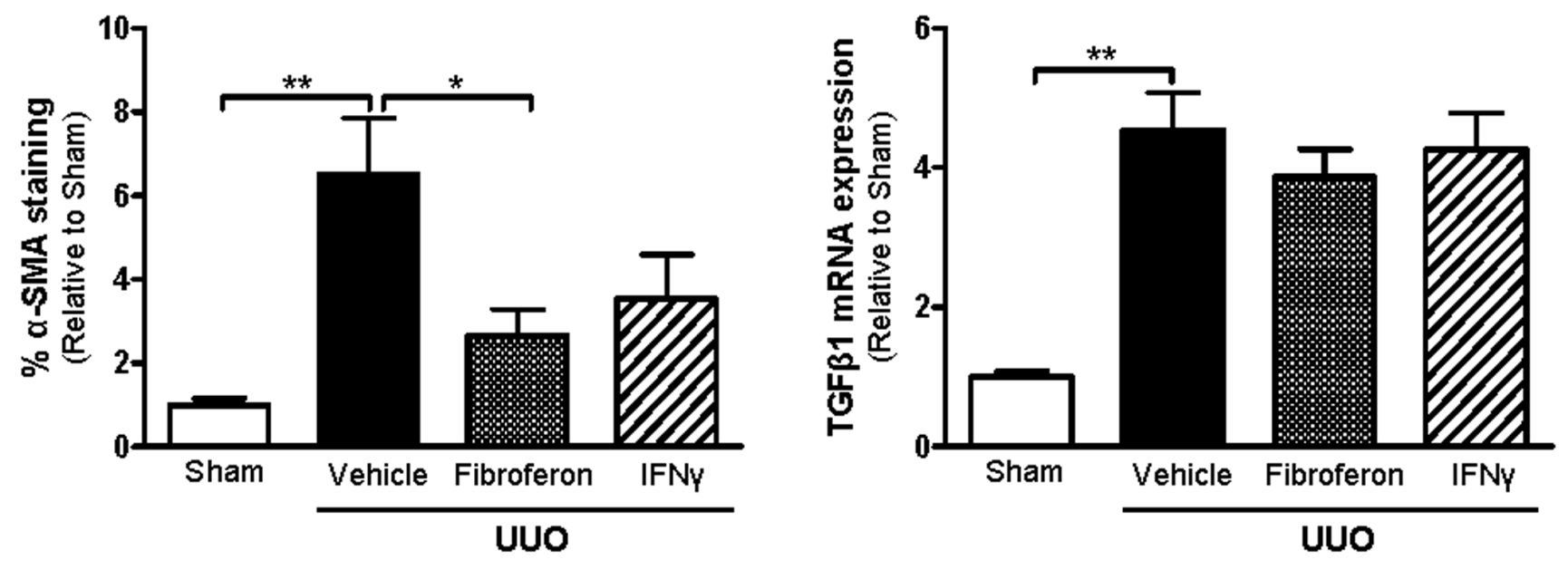

Figure 3: Fibroferon reduces $\alpha$-SMA expression in mouse UUO kidneys. a. Relative gene expression of $\alpha$-SMA in fibrotic UUO and sham-operated control kidneys at day 7 post surgery. b. Representative photomicrographs of $\alpha$-SMA stained kidney sections of sham-operated and UUO mice treated with vehicle (PBS), Fibroferon or (non-targeted full length) IFN $\gamma$ (200x). Scale bar $=120 \mu \mathrm{m}$. c. Computerized quantitative analysis of $\alpha$-SMA protein expression in UUO and sham-operated kidneys. d. Relative gene expression of TGF $\beta 1$ in UUO and sham-operated kidneys. Quantitative data of UUO are expressed relative to sham. ${ }^{*} p \leq 0.05,{ }^{* *} p<0.01,{ }^{* * *} p<0.001$. Bars represent mean \pm SEM of 5-6 mice per group. 
a
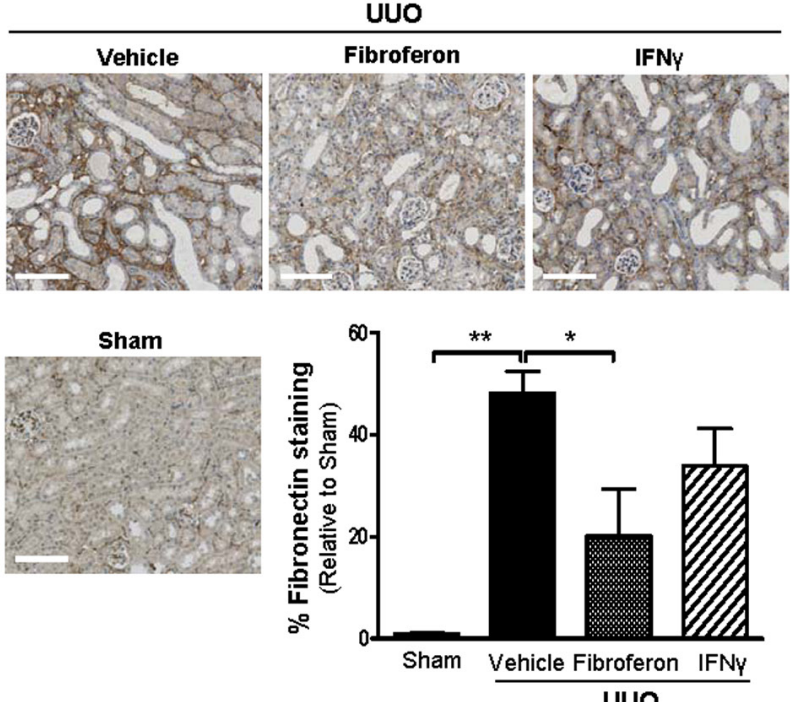

b
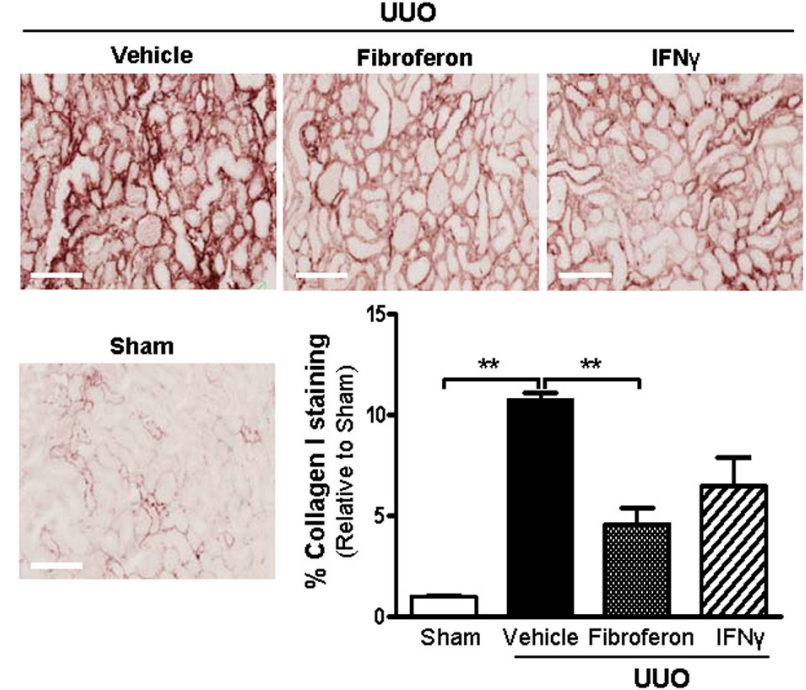

C

UUO
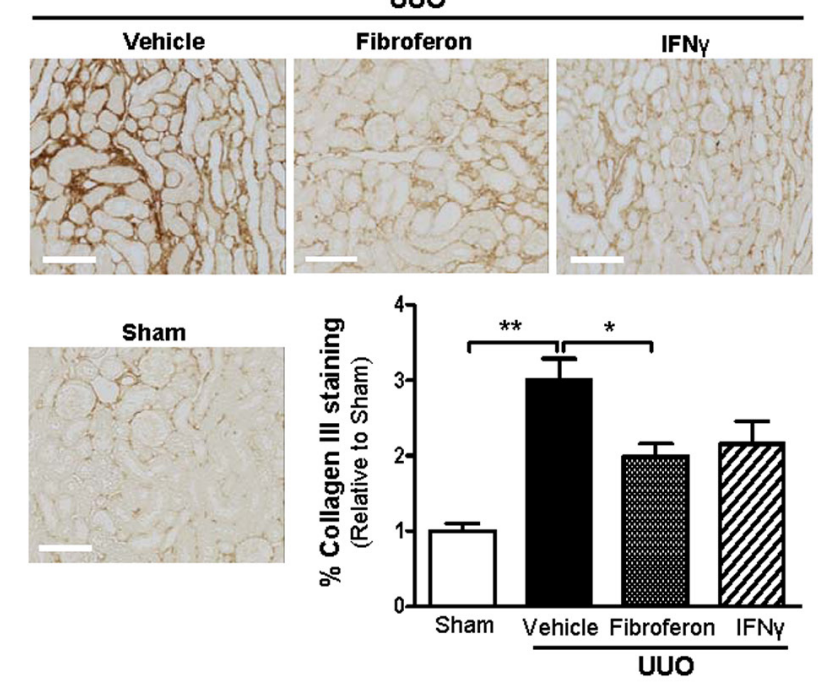

Figure 4: Fibroferon reduces extracellular matrix deposition (fibronectin, collagen I, and collagen III) in mouse UUO kidneys. Representative photomicrographs (200X) and computerized quantitative analysis of a. fibronectin, b. collagen I, and c. collagen III protein expression in UUO and sham-operated kidneys. Scale bar $=120 \mu \mathrm{m}$. Quantitative data of UUO are expressed relative to sham. ${ }^{*} p \leq 0.05,{ }^{* *} p<0.01$. Bars represent mean \pm SEM of 5-6 mice per group. 
side effects in the UUO mouse model of renal fibrosis. This strategy of targeting a chimeric anti-fibrotic biological to PDGF $\beta$ R provides new opportunities for the treatment of renal fibrosis as well as other conditions associated with enhanced PDGF $\beta$ R expression such as cancer and vascular remodeling.

Renal fibrosis is the result of tissue injury and remodeling and is a common hallmark and cause of ESRD [27]. Myofibroblast activation and proliferation play a fundamental role in fibrosis progression [28]. Recent progress in understanding the mechanisms underlying the pathogenesis of fibrosis has led to the expectation that various cytokines such as IFN $\gamma$ could be potentially useful therapeutics to halt or even prevent fibrosis [29]. However, the major obstacle in IFN $\gamma$-based therapy is the manifestation of clinical side effects, which has led to abortion of clinical trials or restricted diverse clinical use of IFN $\gamma[14,15,30,31]$. These adverse effects often preclude administration of anticipated therapeutic effective dose, leading to lack of efficacy in patients. In line with this, we previously showed that systemic delivery of non-targeted full length IFN $\gamma$ in mice results in leukocyte and endothelial cell activation, flu-like symptoms, neurotropic effects, hyperlipidemia, elevated TNF- $\alpha$ and IL-6, inflammation of the central nervous system, and elevated triglyceride levels [19], effects most likely mediated through binding to the widely expressed IFN $\gamma R$. To overcome this hurdle, cell-specific delivery of IFN $\gamma$ provides a new approach to improve its efficacy and to ameliorate potential systemic side effects. To prevent canonical IFN $\gamma$ signaling, we used mim $\gamma$, which lacks the INF $\gamma$ R- binding domain. Mim $\gamma$ targeted to liver stellate cells was recently shown to exert anti-fibrotic activity in vivo $[21,22]$. Here we tested the anti-fibrotic effects of mim $\gamma$ targeted to PDGF $\beta$ R-expressing cells in a model of renal fibrosis. Mim $\gamma$ was directed to interstitial myofibroblasts using a bicyclic PDGF $\beta$ R-recognizing peptide (Bi-PPB). The mim $\gamma$-BiPPB was recently renamed Fibroferon [23].

Interstitial myofibroblasts are characterized by increased expression of PDGF $\beta \mathrm{R}$ in the human and mouse fibrotic kidney [12]. Bi-PPB is a bi-cyclic peptide that binds to the dimeric PDGF $\beta$ R and may thus be used to deliver the mim $\gamma$ peptide to the target cells. To determine its anti-fibrotic effects, we first examined $\alpha$-SMA expression both at the mRNA and protein levels since increased $\alpha$-SMA levels indicate renal myofibroblast activation, the hallmark of fibrogenesis. We indeed observed a clear inhibitory effect of mim $\gamma$-BiPPB on myofibroblast activation (i.e. reduced $\alpha$-SMA expression). ECM composition is significantly altered and expanded during fibrogenesis, which can severely compromise resident cell function (e.g. tubular epithelium) [5, 32]. We therefore analyzed expression of the most prominent ECM components fibronectin, and collagens I and III. Again, a clear pharmacological effect of Fibroferon was noted, and compared to non-targeted full length IFN $\gamma$ the efficacy of Fibroferon in reducing ECM deposition was significantly higher, illustrating the potency of our cell-specific targeting strategy. Of note, although mRNA expression levels of fibronectin and collagens I \& III were lower in Fibroferon-treated mice compared with vehicletreated mice, these differences were not significant (data not shown). These data suggest that the protective effects of Fibroferon are (partly) mediated by enhanced fibrolysis as we demonstrated previously using another interferon- $\gamma$ based conjugate in a model of liver fibrosis [33]. TGFß1 is a pro-fibrotic protein which instigates fibroblast activation and transformation into myofibroblasts, resulting in collagen synthesis and ECM contraction [24]. However, we did not observe altered TGFß1 expression after IFN $\gamma$ or Fibroferon treatment.

Inflammation commonly precedes and accompanies fibrosis [34]. There is evidence for a direct role of T cells in the pathogenesis of renal interstitial fibrosis which can be explained through different mechanisms [35, 36]: (1) direct effects on fibroblast activation and ECM deposition, (2) indirect effects on fibroblasts via induction of a profibrotic phenotype of infiltrating macrophages resulting in fibroblast activation through profibrotic cytokines and growth factors (e.g. PDGF, CTGF, TGFß1), and (3) indirect effects on fibroblasts via tubular epithelial cell activation and subsequent production of cytokines and growth factors that act on fibroblasts. We indeed observed an almost two-fold reduction in the number of infiltrating $\mathrm{T}$ cells after mim $\gamma$-BiPPB treatment, possibly mediated via reduced expression of $\mathrm{T}$ cell chemoattractants that are shown to be produced by myofibroblasts $[37,38]$.

In the setting of renal fibrosis, injured renal cells and inflammatory cells secrete mediators and growth factors that promote angiogenesis and/or lymphangiogenesis [3741]. Since anti-lymphangiogenic effects of IFN $\gamma$ have been reported [42], we analyzed the potential inhibitory effect of Fibroferon on lymphangiogenesis. Renal lymphangiogenesis in UUO kidneys was significantly reduced following Fibroferon treatment, which was not observed after treatment with non-targeted full length IFN $\gamma$. Angiogenesis was also shown to occur during the progression of fibrosis in UUO [43], which was confirmed in our study. A reduction, though non-significant, was observed in the percentage surface area of CD $31^{+}$ peritubular capillaries following Fibroferon treatment. Reduced lymphangiogenesis and angiogenesis most likely reflect indirect effects secondary to the direct anti-fibrotic effects of Fibroferon on myofibroblasts.

Recently we reported on the anti-fibrotic effects of full length IFN $\gamma$ targeted to PDGF $\beta$ R-expressing myofibroblasts in UUO mice [12]. When comparing the anti-fibrotic effects of targeted full length IFN $\gamma$ [12] and Fibroferon, the attenuating effects on $\alpha \mathrm{SMA}$ and ECM constituents are comparable. However, clear differences were observed in favor of Fibroferon with respect to 
a UUO
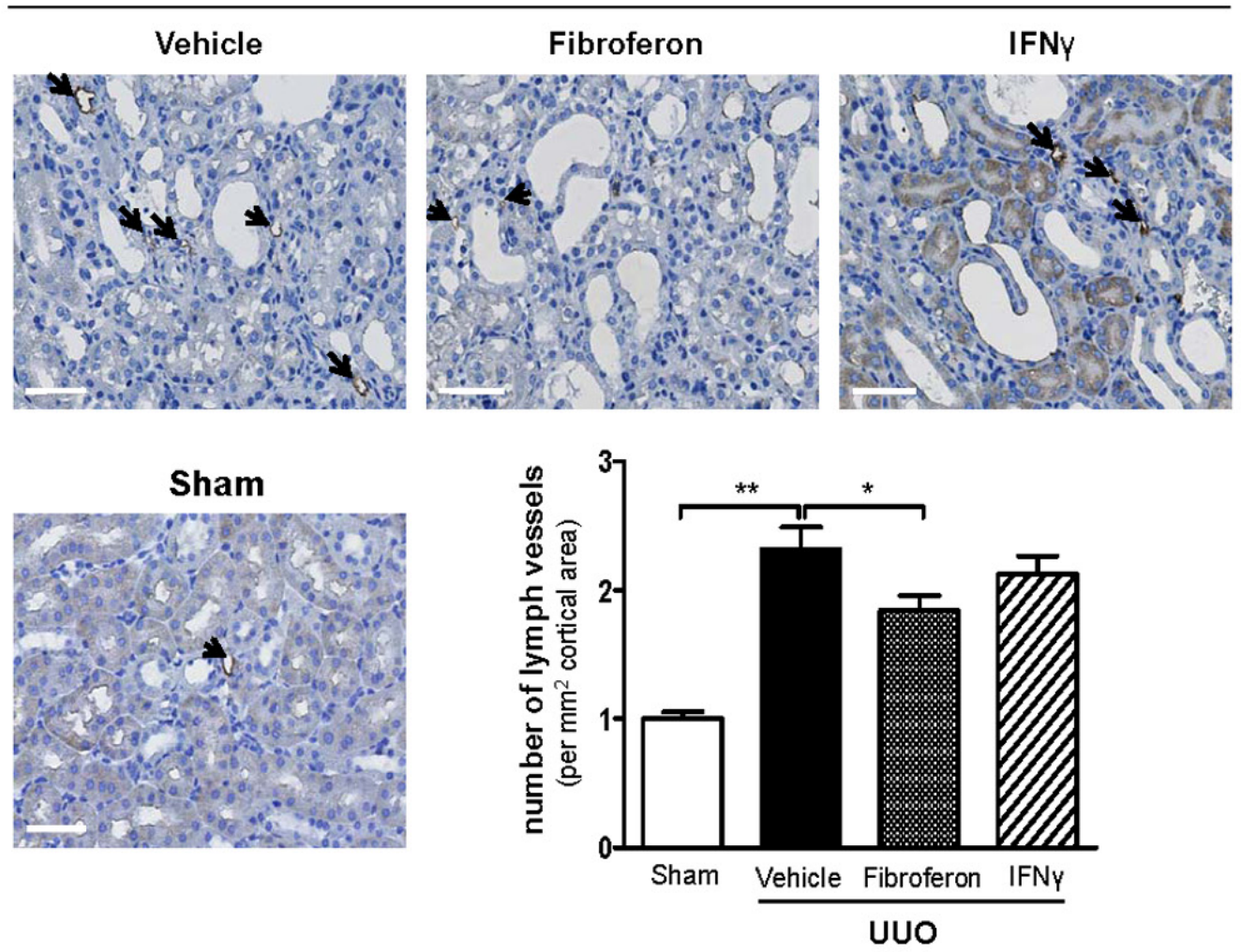

b

UUO
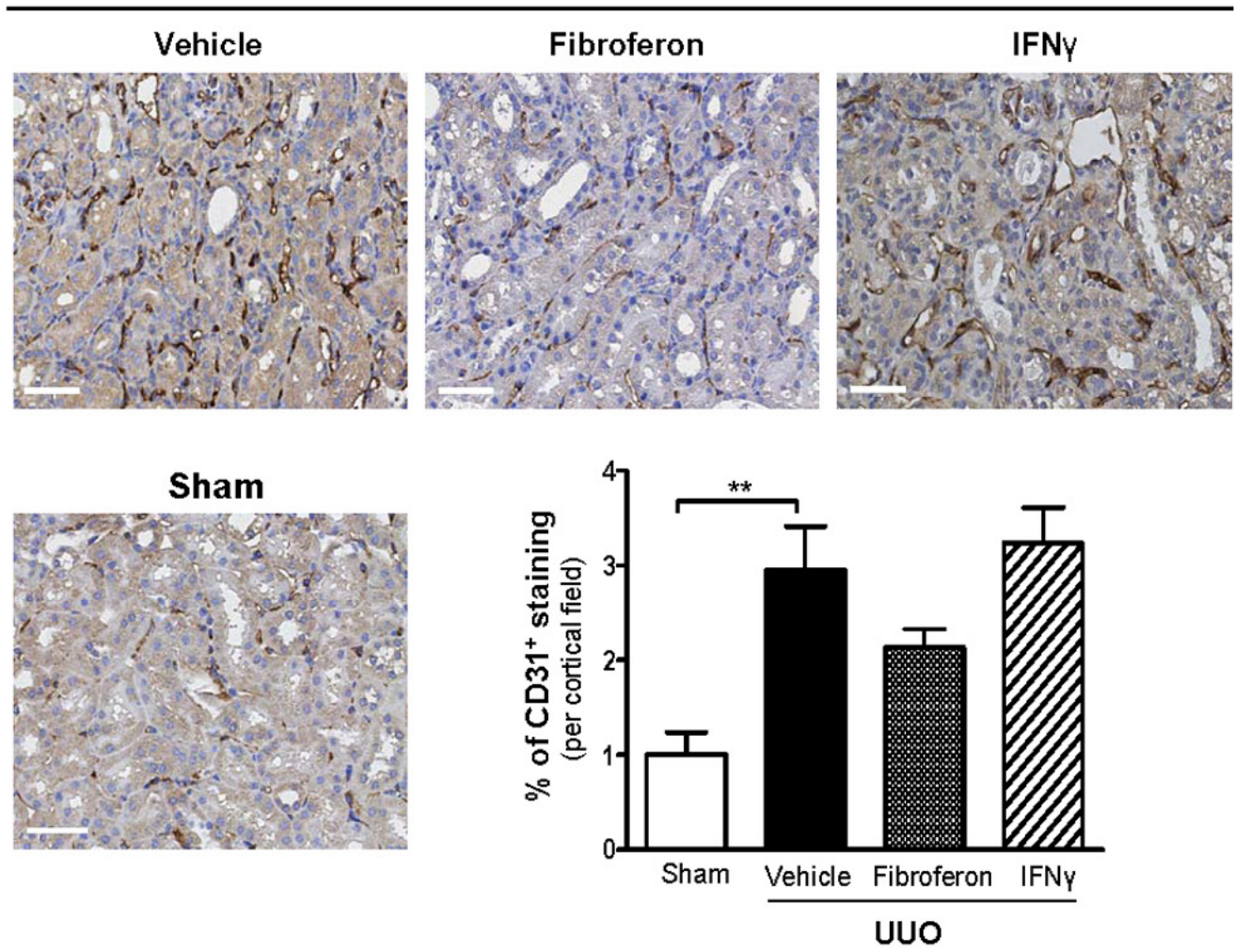

Figure 5: Fibroferon reduces UUO-induced lymphangiogenesis. Representative photomicrographs and quantitative analysis of $\mathbf{a}$. the numbers of podoplanin ${ }^{+}$lymphatic capillaries and $\mathbf{b}$. the numbers of $\mathrm{CD} 31^{+}$peritubular capillaries 7 days post-surgery. Arrows indicate podoplanin $n^{+}$lymphatic capillaries in sham kidney. Scale bar $=50 \mu \mathrm{m}$. Quantitative data of UUO are expressed relative to sham. ${ }^{*} p$ $<0.05,{ }^{* *} p<0.01$. Bars represent mean \pm SEM of 5-6 mice per group. 
CNS and renal inflammation. Compared with targeted full length IFN $\gamma$ [12], brain MHC class II expression was $79 \%$ reduced upon Fibroferon treatment. Similarly, renal F4/80 and MHC class II expression were significantly reduced with $33 \%$ and $53 \%$, respectively. We used MHC class II expression as a marker of macrophage activation. Macrophages are the main target cells for native INF $\gamma$. For anti-fibrotic therapies, generalized macrophage activation is an off-target effect leading to serious adverse effects, and the brain is an off-target organ for renal diseases.
Our data indicate that Fibroferon lacks this off-target effect, in contrast to targeted full length IFN $\gamma$, which can be explained by the lack of Fibroferon binding to the extracellular domain of IFN $\gamma R$. A potential weakness of current study is that we did not include data demonstrating a direct comparison between targeted full length IFN $\gamma$ (PPB-PEG-IFN $\gamma$ ) and Fibroferon. However, we recently published on the anti-fibrotic effects of PPB-PEG-IFN $\gamma$ [12], and, compared with the vehicle control group, the beneficial anti-fibrotic effects of Fibroferon are similar. a

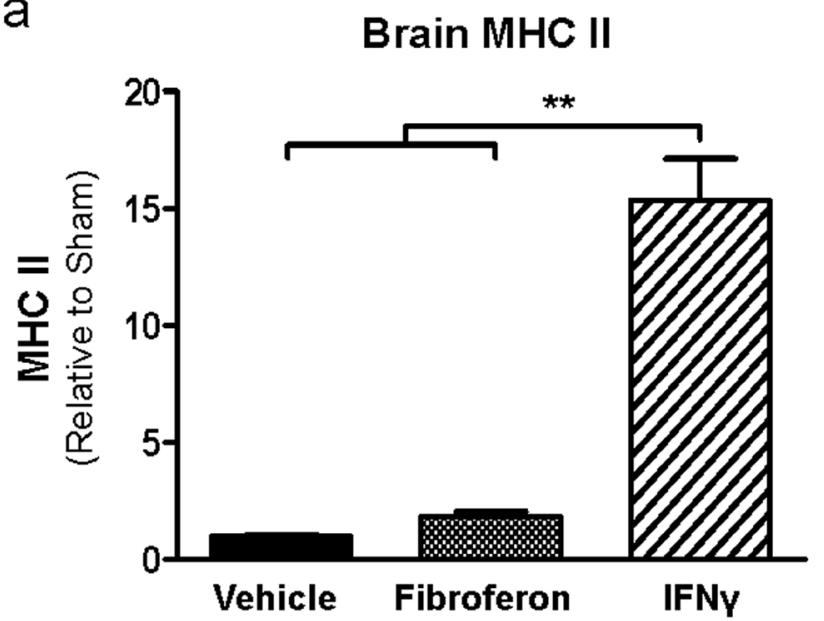

b

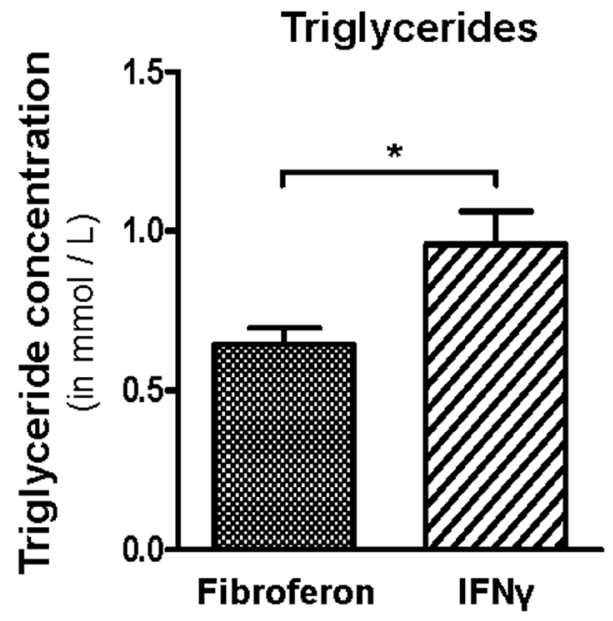

C

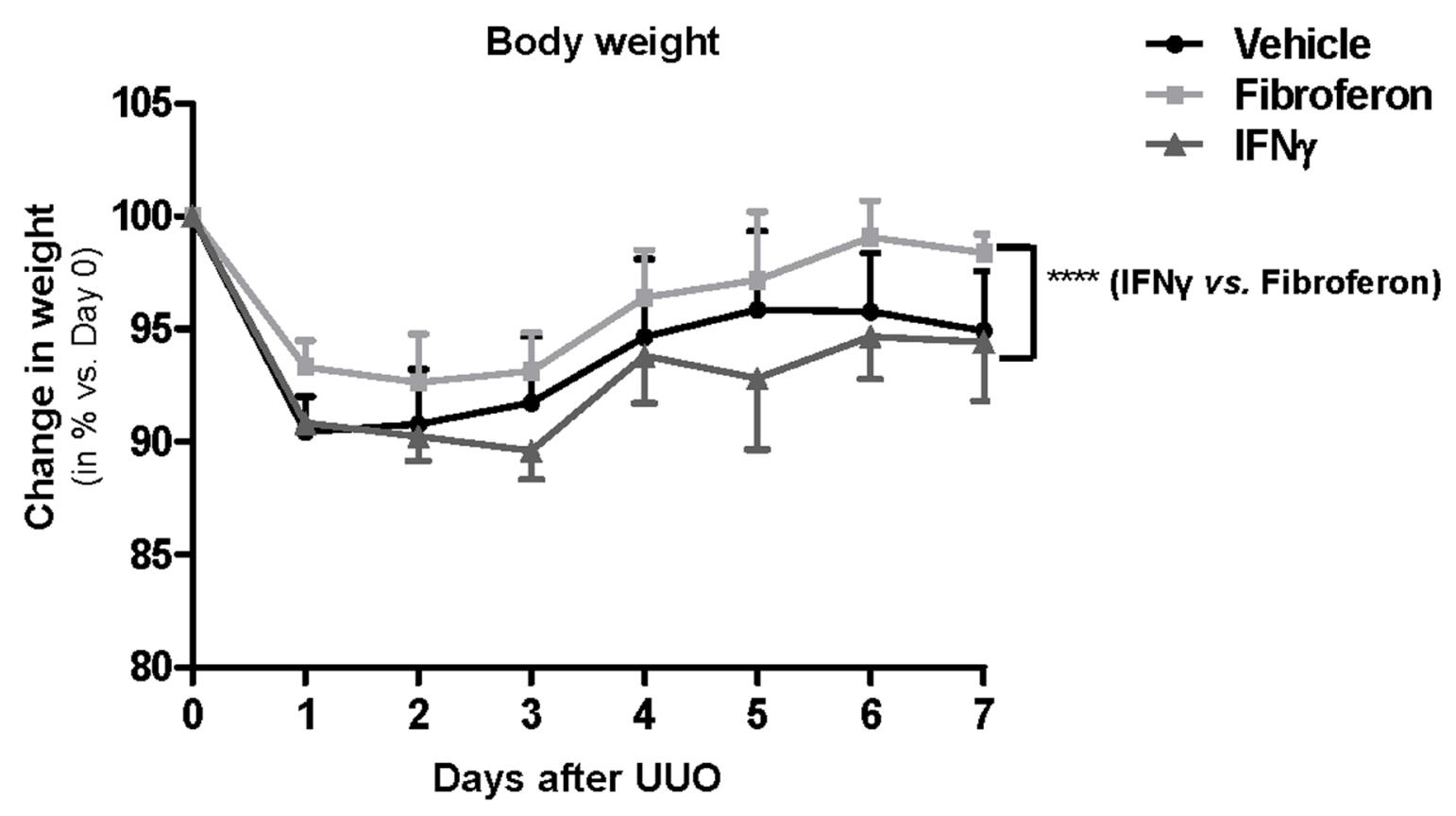

Figure 6: Fibroferon prevents IFN $\gamma$-related systemic side effects and improve weight gain after surgery. a. mRNA expression of brain MHC class II in UUO mice treated with vehicle (PBS), Fibroferon or non-targeted full length IFN $\gamma$ (7 days postsurgery). Data of UUO groups are expressed relative to sham. ${ }^{* *} p<0.01$, Student's t-test. Bars represent mean $\pm \mathrm{SEM}$ of 3 mice per group. b. Plasma triglyceride levels in UUO mice treated with vehicle (PBS), Fibroferon or non-targeted full length IFN $\gamma, 7$ days post-surgery. ${ }^{*} p<$ 0.05 . Bars represent mean \pm SEM of 5-6 mice per group. c. Weight loss and recovery in UUO mice treated with vehicle (PBS), Fibroferon, and (non-targeted full length) IFN $\gamma .{ }^{* * * *} p<0.001$. Change in weight expressed as mean $\pm \mathrm{SD}$ of 6 mice per group/time point. 
The mechanism by which Fibroferon exerts its protective effects is not yet fully elucidated. We previously demonstrated mim $\gamma$ playing a direct role in the activation and nuclear translocation of the transcription factor STAT $1 \alpha$ via the nuclear signaling sequence (NLS) present in the C-terminal region of IFN $\gamma$. This region is able to modulate IFN $\gamma$-responsive genes through activation of the JAK/STAT pathway [21]. Mim $\gamma$ does not recognize the extracellular domain of IFN $\gamma \mathrm{R}$ but rather binds to the cytoplasmic domain of the receptor chain 1 (IFN $\gamma \mathrm{R} 1$ ), and thereby initiates cellular signaling [44-49]. Binding of BiPPB to the PDGF $\beta$ R has been demonstrated in previous studies ${ }^{19,20}$. We propose that $\operatorname{mim} \gamma$-BiPPB binds to the PDGF $\beta$ R and following internalization, the construct is able to interact with the intracellular part of IFN $\gamma \mathrm{R} 1$ and initiates phosphorylation of STAT1 thereby eliciting IFN $\gamma$ related biological effects. Fibroferon just has the IFN $\gamma$ signaling moiety and is only biologically active once it is internalized. This premise needs further study though.

In conclusion, in this study we demonstrate the benefits of (targeted) Fibroferon over non-targeted full length IFN $\gamma$ with respect to its anti-fibrotic effects and the development of off-target systemic side effects. We demonstrate a proof-of-concept strategy for the use of targeted IFN $\gamma$-based biologicals to reduce renal fibrosis by removing the extracellular receptor recognition sequence and directing it to myofibroblasts. The small size and increased stability of Fibroferon compared to the full length targeted IFN $\gamma$ will provide better therapeutic benefits and more possibilities for subcutaneous or muscular administration. In addition, based on these and our previous data we believe that Fibroferon offers high specificity and low toxicity with full agonistic activity, therefore has highly favorable characteristics for the prolonged administration. This strategy is one of the first examples of re-direction of a cytokine to the kidney by creating a chimeric peptide. Our data warrant further exploration of the precise underlying mechanism and mode of action of Fibroferon as well as of the possibility to use Fibroferon to target fibrosis in a clinical setting.

\section{MATERIALS AND METHODS}

\section{Experimental animals and surgical procedures}

Male C57Bl/6 mice (9-12 wks; 28-30 g) were obtained from Harlan (Zeist, the Netherlands). Animals were housed in cages with free access to food and water. During the operation the abdomen was opened by a midline incision under general anesthesia $(2 \%$ isoflurane $/ \mathrm{O}_{2}$ ). All mice were subjected to unilateral ureteral obstruction (UUO) by a double-ligation (using 6-0 silk suture) of the left ureter proximal to the kidney. Right kidneys were used as sham controls and were manipulated but not ligated. After surgery, mice were i.v. injected (penile vein) at days 2, 4 and 6 after surgery (see Figure 1b) with either 1) UUO, vehicle (saline), 2) UUO, Fibroferon (mim $\gamma$-BiPPB, $5 \mu \mathrm{g} /$ mouse/injection), 3) UUO, non-targeted full-length IFN $\gamma$ ( $5 \mu \mathrm{g} /$ mouse/injection). Total follow-up time was 7 days during which mice were weighed daily. Group size was $n=6 /$ group/time point. The experimental animal studies have been approved by the local Institutional Review Board of the University of Groningen (DEC 6066B) and adhered to the national guidelines for the care and use of laboratory animals.

\section{Synthesis of Fibroferon conjugate}

All the chemical reactions were performed in low protein binding tubes (LoBind tubes, Eppendorf, Hamburg, Germany). Bicyclic PDGF $\beta R$-recognizing peptide (CSRNLIDC-GGGDGG-CSRNLIDC with C"C disulfide bonds, BiPPB, $2223 \mathrm{Da}$ ) [18] and peptidomimetic IFN $\gamma$ (mimIFN $\gamma, 4689 \mathrm{Da})$ modified with S-acetyl thioacetate were custom-prepared by Genosphere (Paris, France). To synthesize the construct, BiPPB $(0.112 \mu \mathrm{mol})$ was reacted with $0.337 \mu \mathrm{mol}$ of Maleimide-PEG-succinimidyl carboxy methyl ester (Mal-PEG-SCM, 2 KDa, Creative PEGworks, Winston-Salem, NC) for $3 \mathrm{~h}$. Thereafter, excess of Mal-PEG-SCM was blocked with lysine $(0.337$ $\mu \mathrm{mol})$ for $1 \mathrm{~h}$. Subsequently, the prepared BiPPB-PEGMal was reacted overnight with $\operatorname{mimIFN} \gamma$-ATA in the presence of deacetylating reagent $(0.1 \mathrm{M}$ hydroxylamine, $25 \mathrm{mM}$ EDTA in PBS pH 7.2). The prepared mimIFN $\gamma$ PEG-BiPPB (mim $\gamma$-BiPPB [Fibroferon]) conjugate (8.9 $\mathrm{KDa}$ ) was extensively dialyzed against PBS using $7 \mathrm{kDa}$ dialysis membrane (Thermo Scientific, Rockford, IL).

\section{Antibodies}

For immunohistochemistry the following primary antibodies were used: anti-alpha smooth muscle actin ( $\alpha$-SMA, clone ASM-1, Progen Biotechnik, Heidelberg, Germany); anti-fibronectin (ab6584, Abcam, Cambridge, UK); anti-CD3 (A0452, Dako, Glostrup, Denmark); anti-podoplanin (clone RTD4E10, Acris antibodies, Inc, Herford, Germany.); anti-PDGF $\beta R$ (\#3169, Cell signaling, Danvers, USA), anti-CD31 (clone SZ31, Dianova GmbH, Germany); anti-type I collagen (s131001, SouthernBiotech, USA) and anti-type III collagen (s1330-01, SouthernBiotech, USA).

\section{Immunohistochemistry}

Immunohistochemistry for $\alpha$-SMA, fibronectin, type III collagen, CD31, podoplanin, and CD3 was performed on $2 \mu \mathrm{m}$ paraffin sections. Sections were deparaffinized in xylene and rehydrated in graded alcohol 
series and distilled water. Antigen retrieval was achieved by overnight incubation at $80^{\circ} \mathrm{C}$ in $0.1 \mathrm{M}$ Tris/ $\mathrm{HCl}$ buffer (pH 9.0) for fibronectin and type III collagen staining, and treatment in the microwave for $15 \mathrm{~min}$ at $300 \mathrm{~W}$ with $10 \mathrm{mM}$ citrate buffer $(\mathrm{pH} \mathrm{6:0)}$ for podoplanin and CD31 staining. No antigen retrieval was performed for $\alpha$-SMA staining. Type I collagen and PDGF $\beta$ R staining was performed on $4 \mu \mathrm{m}$ acetone-fixed frozen sections. Endogenous peroxidase activity was blocked with $0.03 \%$ $\mathrm{H}_{2} \mathrm{O}_{2}$ (in PBS) for 30 min. Primary antibody binding was detected by sequential incubations with horseradish peroxidase (HRP)-labeled appropriate secondary and tertiary antibodies (all obtained from DAKO, Glostrup, Denmark). Peroxidase activity was visualized using 3, 3'-diaminobenzidine tetrahydrochloride (DAB, DAKO, Glostrup, Denmark) as chromogen (10 min. incubation). Sections were counterstained with hematoxylin for $1 \mathrm{~min}$ and mounted with Kaiser's glycerin gelatin.

\section{Quantification of immunostaining}

The sections were scanned using a NanoZoomer HT (Hamamatsu Photonics K.K., Shizuoka Pref., Japan). The extent of cortico-interstitial $\alpha$-SMA, fibronectin, type I and III collagens, and CD3 expression was measured as numbers of positive pixels, and the numbers of podoplanin-positive vessel-like structures were counted in 30 cortical areas using Aperio ImageScope software (version 9.1.772.1570, Aperio Technologies Inc, Vista, CA, USA) at 200x magnification. For CD31 staining, 10 cortical areas at 400x magnification were selected, and the percentage of CD31 positive area was measured using Image J 1.46r (Rasband, W.S., U.S. National Institutes of Health).

\section{Quantitative real-time PCR}

Total RNA from kidney tissues was extracted using RNeasy Mini Kit (Qiagen, Hilden, Germany) according to the manufacturer's instructions. The RNA concentrations were measured on a NanoDrop ND-1000 spectrophotometer (Thermo Scientific, USA). Singlestranded cDNA was synthesized using Superscript II and random hexamer primers (Invitrogen, Carlsbad, CA) in a volume of $20 \mu \mathrm{l}$. cDNA's were diluted to a concentration of $2 \mathrm{ng} / \mu \mathrm{l}$ and $2.5 \mu \mathrm{l} /$ reaction ( $5 \mathrm{ng}$ ) was used for qRTPCR analysis. PCR reactions were performed in a $10 \mu \mathrm{l}$ reaction volume containing $1 \mathrm{x}$ qPCR master mix (EUrogentec, Liege, Belgium), 1x Taqman Gene Expression Assay mix (Applied Biosystems, Forster City, CA). The primer reference numbers were as follows: Ywhaz: Mm03950126_s1, $\alpha-S M A / A c t a 2: ~ M m 01546133$ m1, MHC-II/CD74: Mm00658576_m1, and PDGFßR: Mm00435546_m1. qRT-PCR reactions were performed on a ABI7900 HT thermal cycler (Biosystems, Forster
City, CA). Relative gene expression was calculated using the $2^{-\Delta \mathrm{Ct}}$ method with Ywhaz (tyrosine 3-monooxygenase/ tryptophan 5-monooxygenase activation protein $\zeta$ ) as housekeeping gene. Data are expressed as fold-change compared to sham-operated kidneys.

\section{Plasma triglyceride measurements}

Whole blood was collected into commercially available EDTA-coated tubes (Item No: 450474, Greiner Bio-One, GmbH, Austria). The tubes were kept on ice during processing. Cells were removed from plasma by centrifugation for 10 minutes at 1000-2000 x g using a refrigerated centrifuge. Triglyceride levels were measured on a Vitalab Selectra-E chemistry workstation (Vital Scientific BV, Spankeren, The Netherlands).

\section{Statistical analyses}

Statistical analyses were performed using GraphPad Prism 5.0 (GraphPad Software Inc, La Jolla, CA). Differences between multiple groups were calculated using the non-parametric one-way ANOVA (KruskalWallis test) with Dunn's Multiple Comparison Test posttest. Comparison of two groups was performed using the Mann Whitney test (or Student's t-test where indicated). Comparison of weight loss and recovery in the various groups was performed using Repeated Measures ANOVA with Bonferroni post-test. $p \leq 0.05$ was considered statistically significant. Data are expressed as mean \pm standard error of the mean (or mean \pm standard deviation where indicated).

\section{ACKNOWLEDGMENTS}

We thank Catharina Reker-Smit, Eduard Post, Geert Harms, Marian Bulthuis and Sippie Huitema for their technical assistance.

\section{CONFLICTS OF INTEREST}

K.P. is co-founder and shareholder of BiOrion Technologies BV. All other authors declare no competing financial interests.

\section{FUNDING}

F. Poosti was supported by the Graduate School of Medical Sciences (GSMS, University of Groningen, Groningen, The Netherlands), M.H. de Borst was supported by a grant from the Netherlands Organization for Scientific Research (Veni grant). This work was supported by the J.K. de Cock Foundation (University Medical Center Groningen). Microscopical imaging was 
performed at the UMCG Imaging Center (UMIC), which is supported by the Netherlands Organisation for Health Research and Development (ZonMW grant 40-00506-989021).

\section{REFERENCES}

1. Liu Y. Cellular and molecular mechanisms of renal fibrosis. Nat Rev Nephrol. 2011; 7: 684-696.

2. Levey AS, Atkins R, Coresh J, Cohen EP, Collins AJ, Eckardt KU, Nahas ME, Jaber BL, Jadoul M, Levin A, Powe NR, Rossert J, Wheeler DC, et al. Chronic kidney disease as a global public health problem: approaches and initiatives - a position statement from Kidney Disease Improving Global Outcomes. Kidney international. 2007; 72: 247-259.

3. Wynn TA. Common and unique mechanisms regulate fibrosis in various fibroproliferative diseases. The Journal of clinical investigation. 2007; 117: 524-529.

4. Boor P, Ostendorf T, Floege J. Renal fibrosis: novel insights into mechanisms and therapeutic targets. Nat Rev Nephrol. 2010; 6: 643-656.

5. Duffield JS. Cellular and molecular mechanisms in kidney fibrosis. The Journal of clinical investigation. 2014; 124: 2299-2306.

6. LeBleu VS, Taduri G, O'Connell J, Teng Y, Cooke VG, Woda C, Sugimoto H, Kalluri R. Origin and function of myofibroblasts in kidney fibrosis. Nature medicine. 2013; 19: 1047-1053.

7. Meran S, Steadman R. Fibroblasts and myofibroblasts in renal fibrosis. International journal of experimental pathology. 2011; 92: 158-167.

8. Samuel CE. Interferons, Interferon Receptors, Signal Transducer and Transcriptional Activators, and Interferon Regulatory Factors. The Journal of biological chemistry. 2007; 282: 20045-20046.

9. Lee J, Lee J, Park MK, Lim MA, Park EM, Kim EK, Yang EJ, Lee SY, Jhun JY, Park SH, Kim HY, Cho ML. Interferon gamma suppresses collagen-induced arthritis by regulation of Th17 through the induction of indoleamine2,3-deoxygenase. PloS one. 2013; 8: e60900.

10. Yao Y, Zhang J, Tan DQ, Chen XY, Ye DF, Peng JP, Li JT, Zheng YQ, Fang L, Li YK, Fan MX. Interferon-gamma improves renal interstitial fibrosis and decreases intrarenal vascular resistance of hydronephrosis in an animal model. Urology. 2011; 77: 761.e8-761.13.

11. Oldroyd SD, Thomas GL, Gabbiani G, El Nahas AM. Interferon-gamma inhibits experimental renal fibrosis. Kidney international. 1999; 56: 2116-2127.

12. Poosti F, Bansal R, Yazdani S, Prakash J, Post E, Klok $\mathrm{P}$, van den Born J, de Borst MH, van Goor H, Poelstra K, Hillebrands JL. Selective delivery of IFN-gamma to renal interstitial myofibroblasts: a novel strategy for the treatment of renal fibrosis. FASEB journal. 2015; 29: 1029-1042.
13. Gu F, Younes HM, El-Kadi AO, Neufeld RJ, Amsden BG. Sustained interferon-gamma delivery from a photocrosslinked biodegradable elastomer. Journal of controlled release. 2005; 102: 607-617.

14. Cleland JL, Jones AJ. Stable formulations of recombinant human growth hormone and interferon-gamma for microencapsulation in biodegradable microspheres. Pharmaceutical research. 1996; 13: 1464-1475.

15. King TE,Jr, Albera C, Bradford WZ, Costabel U, Hormel P, Lancaster L, Noble PW, Sahn SA, Szwarcberg J, Thomeer M, Valeyre D, du Bois RM, INSPIRE Study Group. Effect of interferon gamma-1b on survival in patients with idiopathic pulmonary fibrosis (INSPIRE): a multicentre, randomised, placebo-controlled trial. Lancet (London, England). 2009; 374: 222-228.

16. Floege J, Eitner F, Alpers CE. A new look at plateletderived growth factor in renal disease. Journal of the American Society of Nephrology. 2008; 19: 12-23.

17. Ostendorf T, Eitner F, Floege J. The PDGF family in renal fibrosis. Pediatric nephrology (Berlin, Germany). 2012; 27: 1041-1050.

18. Beljaars L, Weert B, Geerts A, Meijer DK, Poelstra K. The preferential homing of a platelet derived growth factor receptor-recognizing macromolecule to fibroblast-like cells in fibrotic tissue. Biochemical pharmacology. 2003; 66: 1307-1317.

19. Bansal R, Prakash J, Post E, Beljaars L, Schuppan D, Poelstra K. Novel engineered targeted interferon-gamma blocks hepatic fibrogenesis in mice. Hepatology (Baltimore, Md.). 2011; 54: 586-596.

20. Bansal R, Tomar T, Ostman A, Poelstra K, Prakash J. Selective targeting of interferon gamma to stromal fibroblasts and pericytes as a novel therapeutic approach to inhibit angiogenesis and tumor growth. Molecular cancer therapeutics. 2012; 11: 2419-2428.

21. Bansal R, Prakash J, De Ruiter M, Poelstra K. Interferon gamma peptidomimetic targeted to hepatic stellate cells ameliorates acute and chronic liver fibrosis in vivo. Journal of controlled release. 2014; 179: 18-24.

22. Bansal R, Prakash J, De Ruiter M, Poelstra K. Targeted recombinant fusion proteins of IFNgamma and mimetic IFNgamma with PDGFbetaR bicyclic peptide inhibits liver fibrogenesis in vivo. PloS one. 2014; 9: e89878.

23. van Dijk F, Olinga P, Poelstra K, Beljaars L. Targeted Therapies in Liver Fibrosis: Combining the Best Parts of Platelet-Derived Growth Factor BB and Interferon Gamma. Frontiers in medicine. 2015; 2: 72.

24. Leask A, Abraham DJ. TGF-beta signaling and the fibrotic response. FASEB journal. 2004; 18: 816-827.

25. Yazdani S, Navis G, Hillebrands JL, van Goor H, van den Born J. Lymphangiogenesis in renal diseases: passive bystander or active participant?. Expert reviews in molecular medicine. 2014; 16: e15. 
26. Chade AR. Renal vascular structure and rarefaction. Comprehensive Physiology. 2013; 3: 817-831.

27. Zeisberg M, Neilson EG. Mechanisms of tubulointerstitial fibrosis. Journal of the American Society of Nephrology. 2010; 21: 1819-1834.

28. Strutz F, Zeisberg M. Renal fibroblasts and myofibroblasts in chronic kidney disease. Journal of the American Society of Nephrology. 2006; 17: 2992-2998.

29. Strutz F, Heeg M, Kochsiek T, Siemers G, Zeisberg M, Muller GA. Effects of pentoxifylline, pentifylline and gamma-interferon on proliferation, differentiation, and matrix synthesis of human renal fibroblasts. Nephrology, dialysis, transplantation. 2000; 15: 1535-1546.

30. Boman BM, Gagen MM, Bonnem E, Ajani JA, Schmidt S, Dimery IW, Golando J, Neidhart J. Phase I study of recombinant gamma-interferon (rIFN-gamma). Journal of Biological Response Modifiers. 1988; 7: 438-446.

31. Ziesche R, Hofbauer E, Wittmann K, Petkov V, Block LH. A preliminary study of long-term treatment with interferon gamma-1b and low-dose prednisolone in patients with idiopathic pulmonary fibrosis. The New England journal of medicine. 1999; 341: 1264-1269.

32. Friedman SL, Sheppard D, Duffield JS, Violette S. Therapy for fibrotic diseases: nearing the starting line. Science translational medicine. 2013; 5: 167sr1.

33. Bansal R, Prakash J, de Ruijter M, Beljaars L, Poelstra K. Peptide-modified albumin carrier explored as a novel strategy for a cell-specific delivery of interferon gamma to treat liver fibrosis. Molecular pharmaceutics. 2011; 8: 18991909.

34. Cao Q, Harris DC, Wang Y. Macrophages in kidney injury, inflammation, and fibrosis. Physiology (Bethesda, Md.). 2015; 30: 183-194.

35. Nikolic-Paterson DJ. CD4+ T cells: a potential player in renal fibrosis. Kidney international. 2010; 78: 333-335.

36. Tapmeier TT, Fearn A, Brown K, Chowdhury P, Sacks SH, Sheerin NS, Wong W. Pivotal role of CD4+ T cells in renal fibrosis following ureteric obstruction. Kidney international. 2010; 78: 351-362.

37. Powell DW, Mifflin RC, Valentich JD, Crowe SE, Saada JI, West AB. Myofibroblasts. I. Paracrine cells important in health and disease. The American Journal of Physiology. 1999; 277: C1-9.

38. Gonzalez-Cuadrado S, Bustos C, Ruiz-Ortega M, Ortiz A, Guijarro C, Plaza JJ, Egido J. Expression of leucocyte chemoattractants by interstitial renal fibroblasts: upregulation by drugs associated with interstitial fibrosis. Clinical and experimental immunology. 1996; 106: 518522.
39. Kerjaschki D. The lymphatic vasculature revisited. The Journal of clinical investigation. 2014; 124: 874-877.

40. Sakamoto I, Ito Y, Mizuno M, Suzuki Y, Sawai A, Tanaka A, Maruyama S, Takei Y, Yuzawa Y, Matsuo S. Lymphatic vessels develop during tubulointerstitial fibrosis. Kidney international. 2009; 75: 828-838.

41. Tanaka T, Nangaku M. Angiogenesis and hypoxia in the kidney. Nat Rev Nephrol. 2013; 9: 211-222.

42. Shao X, Liu C. Influence of IFN- alpha and IFN- gamma on lymphangiogenesis. Journal of interferon \& cytokine research. 2006; 26: 568-574.

43. Kida Y, Tchao BN, Yamaguchi I. Peritubular capillary rarefaction: a new therapeutic target in chronic kidney disease. Pediatric nephrology (Berlin, Germany). 2014; 29: 333-342.

44. Ahmed CM, Burkhart MA, Subramaniam PS, Mujtaba MG, Johnson HM. Peptide mimetics of gamma interferon possess antiviral properties against vaccinia virus and other viruses in the presence of poxvirus B8R protein. Journal of virology. 2005; 79: 5632-5639.

45. Ahmed CM, Martin JP, Johnson HM. IFN mimetic as a therapeutic for lethal vaccinia virus infection: possible effects on innate and adaptive immune responses. Journal of immunology (Baltimore, Md.: 1950). 2007; 178: 45764583.

46. Szente BE, Soos JM, Johnson HW. The C-terminus of IFN gamma is sufficient for intracellular function. Biochemical and biophysical research communications. 1994; 203: 16451654.

47. Subramaniam PS, Flowers LO, Haider SM, Johnson HM. Signal transduction mechanism of a peptide mimetic of interferon-gamma. Biochemistry. 2004; 43: 5445-5454.

48. Subramaniam PS, Green MM, Larkin J,3rd, Torres BA, Johnson HM. Nuclear translocation of IFN-gamma is an intrinsic requirement for its biologic activity and can be driven by a heterologous nuclear localization sequence. Journal of interferon \& cytokine research. 2001; 21: 951959.

49. Subramaniam PS, Mujtaba MG, Paddy MR, Johnson HM. The carboxyl terminus of interferon-gamma contains a functional polybasic nuclear localization sequence. The Journal of biological chemistry. 1999; 274: 403-407. 